\title{
GENETICS
}

\section{New miRNA classifier distinguishes CTCL from benign skin disorders}

Cutaneous T-cell lymphomas (CTCL) are the most frequently occurring primary skin lymphoma. These skin lesions contain a high number of inflammatory cells but early diagnosis of CTCL is challenging because of its clinical, pathological and histological similarity to benign inflammatory skin lesions such as psoriasis and eczema. Now, a study has shown that a microRNA (miRNA) classifier can distinguish CTCL from benign skin disorders. Senior researcher Niels Ødum explains the background to the study "we decided to address if a novel miRNA technology could help solve a 100-year-old problem-namely, how to distinguish cutaneous CTCL from benign dermatosis".

The researchers assessed 148 patient samples (63 patients with CTCL and 85 with benign lesions) using microarray miRNA profiling and the classifier with the best performance was confirmed by conventional quantitative reverse transcription (RT) PCR. Using this technology, they identified five miRNAs in the classifier that discriminated between malignant and benign samples with more than $90 \%$ accuracy. The findings were validated in an independent cohort of 50 patients. As Ødum explains, "the most significant finding is that it is possible to distinguish malignant and non-malignant inflammation with a high accuracy by only a few miRNAs: miR-155 is overexpressed and miR-203 and miR-205 are decreased in CTCL. It was very encouraging that the miRNA classifier also worked in early stages of CTCL”. Ødum's team are extending this study to include new independent patient cohorts, and they plan to develop a kit for diagnostic use.

\section{Lisa Hutchinson}

Original article Ralfkiaer, U. et al. Diagnostic microRNA profiling in cutaneous T-cell lymphoma (CTCL). Blood doi:10.1182/blood-2011-06-358382 